\title{
Quaternion-Octonion Analyticity for Abelian and Non-Abelian Gauge Theories of Dyons
}

\author{
P. S. Bisht ${ }^{(1)}$ and O. P. S. Negi ${ }^{(2) *}$
}

October 22, 2018

(1) Department of Physics

Kumaun University

S. S. J. Campus

Almora - 263601 (Uttarakhand) INDIA

(2) Institute of Theoretical Physics

Chinese Academy of Sciences

KITP Building Room No.- 6304

Hai Dian Qu Zhong Guan Chun Dong Lu

55 Hao , Beijing - 100080, P. R. CHINA

\author{
Email: - ps_bisht123@rediffmail.com \\ ops_negi@yahoo.co.in
}

\begin{abstract}
Einstein- Schrödinger (ES) non-symmetric theory has been extended to accommodate the Abelian and non-Abelian gauge theories of dyons in terms of the quaternion-octonion metric realization. Corresponding covariant derivatives for complex, quaternion and octonion spaces in internal gauge groups are shown to describe the consistent field equations and generalized Dirac equation of dyons. It is also shown that quaternion and octonion representations extend the so-called unified theory of gravitation and electromagnetism to the Yang-Mill's fields leading to two $S U(2)$ gauge theories of internal spaces due to the presence of electric and magnetic charges on dyons.

Key words: - Non-symmetric, Quaternion, Octonion, Monopole, Dyons and Gauge theories.

PACS No: - $14.80 \mathrm{Hv}$
\end{abstract}

${ }^{*}$ Permanent Address- Department of Physics, Kumaun University, S. S. J. Campus, Almora -263601 (Uttarakhand) INDIA 


\section{Introduction}

Einstein- Schrödinger (ES) theory [1, 2], a generalization of general relativity, allows a nonsymmetric fundamental tensor and connection. It contains a non symmetric metric whose real symmetric part is described as general relativity while imaginary ( a skew symmetric) part was taken by Einstein [1] as proportional to the electromagnetic tensor. Research in this direction ultimately proved fruitless; the desired classical unified field theory was not found and the interpretation of the skew symmetric part of the metric, as an electromagnetic field tensor, has been shown physically incorrect [3]. However, Moffat [4] and others [5] showed that instead of electromagnetism the anti-symmetric part of the generalized metric tensor represents a kind of non-symmetric gravitational field which is free from ghost poles, tachyons and higher-order poles, and there are no problems with asymptotic boundary conditions. Einstein-Schrödinger (ES) theory has also been modified and extended [6] to include a large cosmological constant (caused by zero-point fluctuations) and sources (spin-0 and spin- $\frac{1}{2}$ ). In the weak field approximation where interaction between fields is not taken into account, the resulting theory is characterized by a symmetric rank-2 tensor field (gravity), an anti-symmetric tensor field, and a constant characterizing the mass of the antisymmetric tensor field. The anti-symmetric tensor field is found to satisfy the equations of a Maxwell-Proca massive anti-symmetric tensor field. Furthermore, the theory permits one or more "running constants": it allows the mass of the anti-symmetric field, the coupling constant between the anti-symmetric field and matter, and the gravitational constant to vary as functions of space and time coordinates. In other words, non-symmetric gravitation theory (NGT) can be described as a theory that involves a symmetric tensor field (gravity), an anti-symmetric tensor field, and one or more scalar fields. On the other hand, Borchsenius [7] developed a principle of correspondence and constructed an unified non-symmetric theory which includes gravity, electromagnetism and Yang Mills field theory. Unfortunately, none of the non-symmetric unified models survived as a plausible theory. Besides the problem of spin-0and not the spin- 1content of the antisymmetric part of the metric, it was shown by Damour et al [8] that the Einstein theory and those which are based on the Einstein Lagrangian, exhibit negative-energy (ghosts) radiative modes and accordingly the Borchsenius [7] theory, which includes the Yang Mills field in Bonor- Moffat- Boal (BMB) [5] theory, has the same problems. However, the inconsistencies and cure as well as problems and hopes have always been challenging issues [8, 9] in non-symmetric gravity theories and still the status of ES or NGT is not clear. Moreover, Morques and Oliveira [10] has developed the quaternionoctonion geometrical extension and interpretation of Einstein- Schrödinger (ES) non-symmetric theory which includes consistently [1] the Bonor- Moffat- Boal (BMB) [5] and Borchsenius [7] theories. It is shown [10, 11] that the real algebra describes general theory of relativity, the complex algebra gives the interpretation of Einstein- Schrödinger (ES) non-symmetric theory and Borchsenius theory [7] is interpreted in terms of quaternions isomorphic to $S U(2)$ group. Similar work has been done by Ragusa [12] by enlarging NGT to include the Yang-Mills field theory and it is shown that the anti-symmetric part of the metric tensor $(2 \times 2$ matrix), describes both types of field equations namely the electromagnetism and Yang-Mills field in the flat space linear approximation. Yet, the inconsistencies and cure as well as problems and hopes have always been challenging issues [12] in non-symmetric gravity theories . On the other hand, despite of the potential importance of monopoles [13] and dyons [14], the formalism necessary to describe them has been clumsy and not manifestly covariant [15, 6]. So, a self consistent and manifestly covariant theory of generalized electromagnetic fields of dyons (particle carrying electric and magnetic charges) has been constructed [17, 18, 19, 20] in terms of two four - potentials 
21] to avoid the use of controversial string variables. The generalized charge, generalized four potential, generalized field tensor, generalized field vector and generalized four - current density associated with dyons have been described as complex quantities with their real and imaginary parts as electric and magnetic constituents.

So, without going in to the controversies of ES or NGT theories, and keeping in mind the potential importance of monopoles (or dyons), in the present paper, we have extended the quaternion-octonion generalization of non-symmetric metric theory developed by Morques et al [10, 11] to accommodate the Abelian and non-Abelian gauge theories of the generalized fields of dyons (particles carrying simultaneous electric and magnetic charges). We have applied here the non-symmetric metric theory and the corresponding affine geometry for three different spaces over the field of complex, quaternion and octonion hyper complex number systems. It has also been shown that the symmetric part of the unified metric theory is associated with gravity while anti-symmetric part is described as the generalized electromagnetic field tensor of dyons. Extension of the metric by quaternionic tangent space has been shown to describe the total curvature in terms of gravitation and electromagnetism, along with the non-Abelian Yang-Mill's field ( internal quaternion curvature) while the further extension of the metric theory to the case of octonions leads the internal octonionic curvature which gives rise to two different Yang-Mill's gauge fields. So, the present theory describes the combined gauge structures $G L(R) \otimes U(1)_{e} \otimes U(1)_{m} \otimes S U(2)_{e} \otimes S U(2)_{m}$ where $G L(R)$ describes Gravity, $U(1)_{e}$ demonstrates the electromagnetism due to the presence of electric charge, $U(1)_{m}$ is responsible for the electromagnetism due to magnetic monopole, $S U(2)_{e}$ demonstrates the Yang Mill's field due to the presence of electric charge while $S U(2)_{m}$ gives rise the another Yang-Mills field due to the presence of magnetic monopole. It has also been shown that this unified picture reproduces the Gravity, electromagnetism and theory of Yang-Mill's field in the absence of magnetic monopole. Accordingly we have obtained the generalized Dirac equation for dyons from the covariant derivatives in terms of complex, quaternionic and octonionic tangent spaces.

\section{Quaternion-Octonion Generalization of Non-Symmetric Metric}

The real formulation of non-symmetric theory is expressed [10, 11] in terms of the real tensor $g_{\mu \nu}$ as,

$$
g_{\mu \nu}=h_{\mu \nu}+k_{\mu \nu}
$$

and its conjugate is accordingly given by

$$
\overline{g_{\mu \nu}}=h_{\mu \nu}-k_{\mu \nu}=h_{\mu \nu}+k_{\mu \nu}=g_{\nu \mu}
$$

So, in Non-Riemannian space - time, ES non - symmetric theory is described in terms of an $n-$ dimensional internal space and thus the line element on the curved space-time is written [10, 11] as

$$
d s^{2}=\frac{1}{n} \operatorname{Tr}\left(G_{\mu \nu} d x^{\mu} d x^{\nu}\right)
$$


where

$$
G_{\mu \nu}=\left(G_{\mu \nu b}^{a}(x)\right), \quad \forall a, b=1,2 \ldots n
$$

is a matrix of internal space such that

$$
\left(\frac{1}{n}\right) \operatorname{Tr} G_{\mu \nu}=g_{\mu \nu}
$$

Here $g_{\mu \nu}$ is the metric of the ES asymmetric theory and $\operatorname{Tr}$ is acting on internal matrices. We have

$$
G_{\mu \nu}^{\dagger}=G_{\nu \mu}
$$

where the $(\dagger)$ operation is used for the Hermitian conjugate. Here $G_{\mu \nu}$ is an object with two matrix indexes $a$ and $b$ in internal space supposedly restricted to the internal space of $2 \times 2$ unitary matrices of $S U(2)$ symmetry group. So, each object in this space may then be written as a linear combination of four linearly independent matrices $\tau_{\mu},(\mu=0,1,2,3)$, where $\tau_{0}=0$ and $\tau_{j}^{\dagger}=\tau_{j} \quad(j=1,2,3)$. Hence the metric (4) may now be written as

$$
G_{\mu \nu}=g_{\mu \nu} \tau_{0}+f_{\mu \nu i} \tau_{i}
$$

with

$$
g_{\mu \nu}=g_{\mu \nu}+i F_{\mu \nu}
$$

where $F_{\mu \nu}$ is the Maxwell's tensor,$f_{\mu \nu i}$ represents the Yang-Mills field strength and for brevity, we have set all the coefficients (or constants) as unity. The internal covariant derivative of a vector (or a spinor in spin space) $\psi^{a}=\psi^{a}(x), \forall a=1,2$, is defined as

$$
\psi_{\| \mu}^{a}=\psi_{, \mu}^{a}+\Gamma_{\mu b}^{a} \psi^{b}
$$

Here the affinity $\Gamma_{\mu}=\left(\Gamma_{\mu b}^{a}(x)\right)$ is the object which makes $\psi_{, \mu}^{a}$ to transform like a vector under transformations in the internal space. $\Gamma_{\mu}$ can be related with the gauge potential as

$$
\Gamma_{\mu}=i C_{\mu} \cdot \tau
$$

and obeys the following internal transformations law as

$$
\Gamma_{\mu}^{\prime}=U(x) \Gamma_{\mu} U^{-1}(x)-\frac{\partial U(x)}{\partial x^{\mu}} U^{-1}(x)
$$


where $U(x)$ is defined in terms of the internal transformation matrices of local $S U(2)$ gauge group. In the curved space-time $\Gamma_{\mu}$, transforms like a vector. Thus the internal curvature is defined as

$$
\psi_{\| \mu \nu}^{a}-\psi_{\| \nu \mu}^{a}=P_{\mu \nu b}^{a} \psi^{b}
$$

Here $P_{\mu \nu b}^{a}$ is the curvature in the internal space, i.e.

$$
\begin{gathered}
P_{\mu \nu}=\Gamma_{\mu, \nu}-\Gamma_{\nu, \mu}-\left[\Gamma_{\mu}, \Gamma_{\nu}\right] \\
P_{\mu \nu}=-P_{\nu \mu}
\end{gathered}
$$

In case of complex tangent space, the $U(x)$ "internal transformation matrix" is described by the matrices of the internal gauge group $U(1)$. Hence the transformation laws of an object in the complex $C$ - space, $K$ may be written as,

$$
K^{\prime}=U(1) K
$$

where $U(1)$ stands for a unitary $1 \times 1$ (local) transformation matrix, $U(1)=e^{i \phi(x)}$, and

$$
\bar{K}^{\prime}=\bar{U}(1) \bar{K}
$$

where $\bar{U}(1)=U^{-1}(1)=e^{-i \phi(x)}$. Accordingly, the "internal connection" $C_{\nu}$ is transformed as

$$
C_{\nu}^{\prime}=U(1) C_{\nu} U^{-1}(1)-U_{, \nu}(1) U^{-1}(1)
$$

It should be noted that the connection $C_{\nu}$ transforms as a vector under space - time transformations. In a particular case where the internal transformations are represented by the matrices $U(1)=1+i \phi$, the connection $C_{\nu}$ transforms in first order as,

$$
C_{\nu}^{\prime}=C_{\nu}+i \phi_{, \nu}
$$

which follows the gauge transformation law for an electromagnetic potential.

In the Borchsenius theory [7] , the vector space is described in terms of Pauli matrices which can be reinterpreted as quaternions [10, 11] to describe quaternion tangent space. In this case, we take $e_{j}=i^{-1} \sigma_{j}, \forall j=1,2,3 ; i=\sqrt{-1} ; \sigma_{i}$ are Pauli matrices and $e_{j}$ are quaternion basis elements satisfying the following multiplication relation

$$
e_{j} e_{k}=-\delta_{j k} e_{0}+\varepsilon_{j k l} e_{k}
$$

where $e_{0}$ is the unit element of the quaternion algebra i.e. $e_{0}=\sigma_{0}$ and $\delta_{j k}$ is the Kronecker 
delta symbol. So, the metric in quaternionic space - time undergoes with the symmetry property given by equation (6) where the Hermitian conjugation operation is carried out in terms of the quaternionic internal space or $Q-$ space and $\Gamma_{\nu}=-C_{\nu}^{a} e_{a}$ is the affinity in the quaternionic internal space and transforms under the transformation laws given by equation (11). Since, quaternion basis elements are isomorphic to the algebra of Pauli spin matrices, we may obtain other results given by equations (12, 13) and (14) in quaternion tangent space.

For octonion tangent space, we use the split octonion $O$ algebras where an octonion $P$ is written $[22,23,24]$ in the split $O$ algebra as,

$$
P=a u_{0}^{\star}+b u_{0}-n_{k} u_{k}^{\star}+m_{k} u_{k} \quad \forall k=1,2,3
$$

where $u_{0}^{\star}, u_{0}, u_{k}, u_{k}^{\star}(\forall k=1,2,3)$ are the the split $O$ basis elements [10, 11, 22, 23, 24] defined as

$$
\begin{aligned}
& u_{0}=\frac{1}{2} \quad\left(e_{0}+i e_{7}\right) ; \quad u_{0}^{\star}=\frac{1}{2}\left(e_{0}-i e_{7}\right) ; \\
& u_{k}=\frac{1}{2} \quad\left(e_{k}+i e_{k+3}\right) ; \quad u_{k}^{\star}=\frac{1}{2}\left(e_{k}-i e_{k+3}\right) .
\end{aligned}
$$

Here the set of octets $e_{0}, e_{1}, e_{2}, e_{3}, e_{4}, e_{5}, e_{6}, e_{7}$ are known as the octonion units satisfying the following multiplication rule

$$
e_{0}^{2}=e_{0}=1 ; \quad e_{0} e_{A}=e_{A} e_{0} \quad=e_{A} ; e_{A} e_{B}=-\delta_{A B} e_{0}+f_{A B C} e_{C}(\forall A, B, C=1,2 \ldots .6,7) ;
$$

where the structure constants $f_{A B C}$ is completely antisymmetric and takes the value 1 for following combinations

$$
f_{A B C}=1 \quad \forall(A B C)=(123) ;(471) ;(257) ;(165) ;(624) ;(543) ;(736) .
$$

So, an split octonion $P$ given by equation (20) is now be written [10, 11, 22, 23, 24], in terms of $2 \times 2$ Zorn's vector matrix realizations as .

$$
P \cong Z(P)=\left(\begin{array}{cc}
a & -\vec{n} \\
\vec{m} & b
\end{array}\right) .
$$

We may also express split octonion algebra in terms of Pauli matrices which are related with the quaternion basis elements given by equation (19). So, we define the following $2 \times 2$ Zorn's vector matrix realizations of split octonion basis elements $u_{0}^{\star}, u_{0}, u_{k}, u_{k}^{\star}(\forall k=1,2,3)$ i.e.

$$
\begin{array}{ll}
Z\left(u_{0}^{\star}\right)=\left(\begin{array}{cc}
1 . e_{0} & 0_{2} \\
0_{2} & 0_{2}
\end{array}\right) ; & Z\left(u_{0}\right)=\left(\begin{array}{cc}
0_{2} & 0_{2} \\
0_{2} & 1 . e_{0}
\end{array}\right), \\
Z\left(u_{k}^{\star}\right)=\left(\begin{array}{cc}
0_{2} & -1 . e_{k} \\
0_{2} & 0_{2}
\end{array}\right) ; & Z\left(u_{k}\right)=\left(\begin{array}{cc}
0_{2} & 0_{2} \\
1 . e_{k} & 0_{2}
\end{array}\right) .
\end{array}
$$

The octonion conjugation of $P$ is now defined as 


$$
\bar{P}=b u_{0}^{\star}+a u_{0}+n_{k} u_{k}^{\star}-m_{k} u_{k}^{\star} \quad(\forall k=1,2,3)
$$

and a Hermitian conjugate of $P$ is expressed as

$$
P^{\dagger}=(\bar{P})^{\star}=b^{\star} u_{0}^{\star}+a^{\star} u_{0}+n_{k}^{\star} u_{k}^{\star}-m_{k}^{\star} u_{k}(\forall k=1,2,3) .
$$

As such, we may reformulate equation (44) as the $O$ "metric" with its split form [10, 11] as

$$
G_{\mu \nu}(x)=\left(\begin{array}{cc}
s_{\mu \nu}^{0} e_{0} & -s_{\mu \nu}^{k} e_{\kappa} \\
r_{\mu \nu}^{k} e_{\kappa} & r_{\mu \nu}^{0} e_{0}
\end{array}\right)=G_{\mu \nu}(s, r) .
$$

Here $r_{\mu \nu}^{0}=s_{\mu \nu}^{0}=g_{(\mu \nu)}+i F_{[\mu \nu]} ; g_{(\mu \nu)}$ is identified as the symmetric metric (gravity-expressed in terms of algebra of real numbers $G L(R))$ and $F_{[\mu \nu]}$ is the Maxwell $U(1)$ valued electromagnetic field strength, while $r_{\mu \nu}^{k}$ and $s_{\mu \nu}^{k}$ are $S U(2)$ valued field strengths of two Yang-Mills (non-Abelian gauge) fields. So, we get the following symmetry property

$$
G_{\mu \nu}^{\dagger}(s, r)=G_{\nu \mu}(s, r) .
$$

and

$$
G_{\mu \alpha}(s, r) G^{\mu \nu}(s, r)=G^{\nu \mu}(s, r) G_{\alpha \mu}(s, r)=\delta_{\alpha}^{\nu}\left(u_{0}+u_{0}^{*}\right) .
$$

Here we agree with the statement of Castro [24] that the most salient feature of the split octonion metric $G_{\mu \nu}$ given by equation (28) is that it includes the ordinary space time metric $g_{\mu \nu}$, in addition to electromagnetism and Yang Mills fields. Hence it automatically justifies the KaluzaKlein theory without introducing extra space-time dimensions. The line element in the $O$ space - time is thus defined by

$$
d s^{2}=\frac{1}{4} \operatorname{Tr}\left(d x^{\mu} d x^{\nu} G_{\mu \nu}\right)
$$

while the affinity $\Gamma_{\nu}$ given by equation (9) is expressed in the internal octonionic space as

$$
\Gamma_{\nu}=\left(\begin{array}{cc}
0_{2} & -L_{\nu} \cdot e \\
K_{\nu} \cdot e & 0_{2}
\end{array}\right)
$$

where $\left\{L_{\nu}\right\}$ and $\left\{K_{\nu}\right\}$ are two real four- potentials (gauge connections) analogous to $\left\{C_{\mu}\right\}$ given by equation (10) and discussed above for the quaternionic case. Like equation (13), the octonion curvature $S_{\nu \gamma}$ may then be written as

$$
S_{\nu \gamma c}^{a}=S_{\nu \gamma c}^{a}\left(u_{0}+u_{0}^{*}\right)+\delta_{c}^{a} P_{\nu \gamma}
$$




\section{Non-Symmetric Metric and Dyonic Fields}

Let us extend the Einstein-Schrödinger non -symmetrical metric in terms of three different tangent spaces namely complex, quaternionic and octonionic cases associated with the generalized fields of dyons.

\subsection{Complex Case}

In the complex tangent space case, the non-symmetric metric $g_{\mu \nu}$ given by equation (1) is rewritten as

$$
g_{\mu \nu}=g_{\mu \nu}+i k_{\mu \nu} .
$$

where we represent $F_{[\mu \nu]}=k_{\mu \nu}$ as the anti - symmetric tensor comprising electromagnetic field associated with dyons in the following manner

$$
k_{\mu \nu} \rightarrow\left(F_{\mu \nu}+i F_{\mu \nu}^{d}\right)
$$

where $\left\{F_{\mu \nu}\right\}$ and $\left\{F_{\mu \nu}^{d}\right\}$ are described as generalized electromagnetic and dual electromagnetic fields of dyons [17, 18, 22]. So this extension accommodates both types of non-symmetric metrics real as well as complex where one of the Maxwell field is always real. Thus for the dyonic case, we may identify the internal connection (i.e. $C_{\nu}$ ) as the generalized electromagnetic potential $\left\{V_{\mu}\right\}$ of dyons [17, 18, 22] described as ;

$$
\left\{V_{\mu}\right\}=\left\{A_{\mu}\right\}-i\left\{B_{\mu}\right\} .
$$

Hence, in complex case, non - symmetric metric (35) is associated with our generalized electromagnetic field tensor of dyons [17, 18, 22] (i.e. the matrix of the internal space for dyonic fields) as

$$
\begin{aligned}
G_{\mu \nu} & =F_{\mu \nu}-i F_{\mu \nu}^{d}, \\
G_{\mu \nu}^{\star} & =F_{\mu \nu}+i F_{\mu \nu}^{d}
\end{aligned}
$$

where $(\star)$ denotes the complex conjugation. Hence, replacing the gauge connection $\left\{C_{v}\right\}$ by our generalized four potential $\left\{V_{\nu}\right\}$, we get,

$$
\begin{aligned}
& G_{\mu \nu}=V_{\mu, \nu}-V_{\nu, \mu}, \\
& G_{\mu \nu}^{\star}=V_{\mu, \nu}^{\star}-V_{\nu, \mu}^{\star} .
\end{aligned}
$$

As such, correspondingly, we have the following field equations ,

$$
\begin{aligned}
G_{\mu \nu, \nu} & =J_{\mu}, \\
G_{\mu \nu, \nu}^{\star} & =J_{\mu}^{\star}
\end{aligned}
$$


where $\left\{J_{\mu}\right\}$ represents the generalized current for the dyonic fields given by $\left\{J_{\mu}\right\}=\left\{j_{\mu}^{e}\right\}-i\left\{j_{\mu}^{m}\right\}$ with $\left\{j_{\mu}^{e}\right\}$ and $\left\{j_{\mu}^{m}\right\}$ are described as the four currents respectively associated with electric and magnetic charges. Equation (37) gives the following decompositions of electric and magnetic field strengths of dyons i.e.

$$
\begin{aligned}
& F_{\mu \nu}=\frac{1}{2}\left(G_{\mu \nu}+G_{\mu \nu}^{\star}\right), \\
& F_{\mu \nu}^{d}=-\frac{1}{2 i}\left(G_{\mu \nu}-G_{\mu \nu}^{\star}\right) .
\end{aligned}
$$

Thus we obtain the following decoupled Generalized Dirac-Maxwell's (GDM) equations of dyons in terms of electric and magnetic four currents as

$$
\begin{aligned}
& F_{\mu \nu, \nu}=\frac{1}{2}\left(G_{\mu \nu, \nu}+G_{\mu \nu, \nu}^{\star}\right)=\frac{1}{2}\left(J_{\mu}+J_{\mu}^{\star}\right)=j_{\mu}^{e}, \\
& F_{\mu \nu, \nu}^{d}=-\frac{1}{2 i}\left(G_{\mu \nu, \nu}-G_{\mu \nu, \nu}^{\star}\right)=\frac{1}{2}\left(J_{\mu}-J_{\mu}^{\star}\right)=j_{\mu}^{m}
\end{aligned}
$$

Here dyons are considered as the point particle carrying simultaneous existence of electric and magnetic charges in terms of two Abelian $U(1)$ gauge structures. Now replacing internal connection $\left\{C_{\mu}\right\}$ by generalized potential $\left\{V_{\mu}\right\}$, we may apply the following internal transformation law (22]23) as

$$
V_{\mu} \rightarrow \Omega V_{\mu}^{\star} \Omega^{-1}-\left(\partial_{\mu} \Omega\right) \Omega^{-1}
$$

and the corresponding equation (18) for the dyonic field is then expressed as

$$
V_{\mu} \quad \rightarrow \quad V_{\mu}+i \phi_{, \mu}
$$

which is the gauge transformation for the generalized electromagnetic potential of dyons. Similarly we may write

$$
\begin{array}{lll}
V_{\mu}^{\star} & \rightarrow & \Omega V_{\mu}^{\star} \Omega^{-1}-\left(\partial_{\mu} \Omega\right) \Omega^{-1} \\
\rightarrow & V_{\mu}^{\star} & +i \phi_{, \mu}^{\star}
\end{array}
$$

and consequently we get the following decoupled electric and magnetic $U(1)$ gauge connections

$$
\begin{aligned}
A_{\mu} & =\frac{1}{2}\left(V_{\mu}+V_{\mu}^{\star}\right), \\
B_{\mu} & =\frac{i}{2}\left(V_{\mu}-V_{\mu}^{\star}\right) .
\end{aligned}
$$

Here $A_{\mu}$ and $B_{\mu}$ represent the electric and magnetic four - potential of dyonic fields and are the out comes of the non-symmetric metric in the complex tangent space. As such we may write the covariant derivative $D_{\mu}$ as 


$$
\begin{aligned}
& D_{\mu} \rightarrow \partial_{\mu}+i V_{\mu}, \\
& D_{\nu} \rightarrow \partial_{\nu}+i V_{\nu}
\end{aligned}
$$

and therefore

$$
\left[D_{\mu}, D_{\nu}\right]=D_{\mu} D_{\nu}-D_{\nu} D_{\mu}=G_{\mu \nu}
$$

which satisfies the generalized Maxwell's - Dirac equation for dyonic fields given equation [39, As such, without disturbing the real part of the non symmetric ES metric ( taking it as gravity) we have successfully extended its imaginary part corresponding to the generalized fields of dyons in order to reformulate the self-consistent and manifestly covariant theory of dyons.

\subsection{Quaternion Case}

In order to develop unified quaternionic non - symmetric metric theory, we use the bi quaternionic formulation of dyons described earlier ( Shalini Bisht et al [20] ) instead of using the metric of the real quaternionic tangent space since bi -quaternions work over the filed of complex

numbers like ordinary quaternions do with real numbers. So, the metric given equations (78) is now written as

$$
G_{\mu \nu}=G_{\mu \nu}^{0} e_{0}+G_{\mu \nu}^{j} e_{j}
$$

and

$$
\begin{aligned}
& G_{\mu \nu}^{0}=g_{\mu \nu} \Rightarrow g_{\mu \nu}+i k_{\mu \nu} \Rightarrow g_{\mu \nu}+i\left(F_{\mu \nu}-i F_{\mu \nu}^{d}\right) \\
& G_{\mu \nu}^{j} \Rightarrow f_{\mu \nu j}=f_{\mu \nu j}^{e}-i f_{\mu \nu j}^{m}
\end{aligned}
$$

where superscript $(e)$ and $(m)$ are used for electric and magnetic counter parts of dyons. Accordingly, we may use the properties of quaternion metric, internal covariant derivative, the transformation law, curvature etc. for the quaternionic space-time given by equations (9) to (14).

Let us now define the the covariant derivative [18] in quaternionic non - symmetric metric theory of dyonic fields as

$$
\begin{aligned}
& D_{\mu} \rightarrow \partial_{\mu}+V_{\mu} e_{0}+V_{\mu}^{a} e_{a} \\
& D_{\nu} \rightarrow \partial_{\nu}+V_{\nu} e_{0}+V_{\nu}^{a} e_{a} \quad(a=1,2,3)
\end{aligned}
$$

which gives the complex Abelian and non-Abelian $U(1) \times S U(2)$ gauge structure. The second term in the right hand side of equation (50) represents the electromagnetic $U(1)$ part while the third term represents the non - Abelian $S U(2)$ part of Yang - Mill's field spanned in the term of quaternion basis elements. Then we get 


$$
\left[D_{\mu}, D_{\nu}\right] \psi=\left(D_{\mu} D_{\nu}-D_{\nu} D_{\mu}\right) \psi=\left(G_{\mu \nu} e_{0}+G_{\mu \nu}^{a} e_{a}\right) \psi
$$

which describes $U(1) \times S U(2)$ gauge field strengths for generalized fields of dyons. In equation (51) we have

$$
G_{\mu \nu}=\partial_{\mu} V_{\nu}-\partial_{\nu} V_{\mu}, \quad G_{\mu \nu}^{a}=\partial_{\mu} V_{\nu}^{a}-\partial_{\nu} V_{\mu}^{a}
$$

and subsequently we get the following field equations

$$
G_{\mu \nu, \nu}=D_{\nu} G_{\mu \nu}=j_{\mu} ; \quad G_{\mu \nu, \nu}^{a}=D_{\nu} G_{\mu \nu}^{a}=j_{\mu}^{a} ;
$$

where $j_{\mu}$ and $j_{\mu}^{a}$ are the generalized current corresponding to the electromagnetic part $U(1)$ and non - Abelian part $S U(2)$ respectively for the dyonic fields. So, we get the following continuity equation for generalized fields of dyons as

$$
\partial^{\mu} J_{\mu}=0
$$

but for non-Abelian gauge fields, we get

$$
\partial^{\mu} J_{\mu}^{a} \neq 0 ; \quad D^{\mu} J_{\mu}=0
$$

where

$$
J_{\mu}=J_{\mu} e_{0}+J_{\mu}^{a} e_{a}
$$

which is the $U(1) \times S U(2)$ gauge structure of the generalized current associated with dyons consisting point like $U(1)$ gauge structure of Abelian four current $\left\{J_{\mu}\right\}$ followed by $S U(2)$ like extended Yang - Mill's gauge structure $\left\{J_{\mu}^{a}\right\}$ as the non - Abelian gauge current.

\subsection{Octonion Case}

Octonionic tangent space has been defined in terms of its split basis. Its metric is also defined in split form by equations (28) while line element in the $O$ space - time is expressed by equations (31) and other properties are given by equations (32 33). Octonionic gauge formulation of dyonic fields has also been discussed by us ( Shalini Dangwal et al [22] ). As such, we may straight forwardly write the covariant derivative for the dyonic fields in split octonion form as,

$$
D_{\mu} \rightarrow\left(\begin{array}{cc}
\partial_{\mu}+V_{\mu} & -V_{\mu}^{a} e_{a} \\
V_{\mu}^{a *} e_{a} & \partial_{\mu}+V_{\mu}^{a}
\end{array}\right) ; \quad D_{\nu} \rightarrow\left(\begin{array}{cc}
\partial_{\nu}+V_{\nu} & -V_{\nu}^{a} e_{a} \\
V_{\nu}^{a *} e_{a} & \partial_{\nu}+V_{\nu}^{a}
\end{array}\right)
$$

Then we get 


$$
\left[D_{\mu}, D_{\nu}\right]=\left(\begin{array}{cc}
F_{\mu \nu} & -\overrightarrow{F_{\mu \nu}^{a}} \cdot \overrightarrow{e_{a}} \\
\overrightarrow{f_{\mu \nu}^{a}} \cdot \overrightarrow{e_{a}} & f_{\mu \nu}
\end{array}\right)=G_{\mu \nu}
$$

where

$$
\begin{aligned}
F_{\mu \nu} & =\partial_{\mu} V_{\nu}-\partial_{\nu} V_{\mu}, \\
F_{\mu \nu}^{a} & =\partial_{\mu} V_{\nu}^{a}-\partial_{\nu} V_{\mu}^{a}+i \varepsilon_{a b c} V_{\mu}^{b} V_{\nu}^{c}, \\
f_{\mu \nu} & =\partial_{\mu} V_{\nu}^{\star}-\partial_{\nu} V_{\mu}^{\star}, \\
f_{\mu \nu}^{a} & =\partial_{\mu} V_{\nu}^{a \star}-\partial_{\nu} V_{\mu}^{a \star}+i \varepsilon_{a b c} V_{\mu}^{b \star} V_{\nu}^{c \star}
\end{aligned}
$$

Therefore we may obtain the following split form of field equation as

$$
D_{\mu} G_{\mu \nu}=\left(\begin{array}{cc}
j_{\nu} & -j_{\nu}^{a} e_{a} \\
k_{\nu}^{a} e_{a} & k_{\nu}
\end{array}\right)=J_{\nu}
$$

and the $U(1) \times S U(2)$ form of generalized continuity equation as

$$
D_{\nu} J_{\nu}=0 .
$$

as such, the octonion extension of unified non-symmetric metric for the case of dyons is described in terms two $U(1)$ Abelian ( electromagnetic) and two $S U(2)$ non Abelian (Yang-Mills field). Thus for the case of quaternions and octonions we need not to define the Yang Mills field by hand. The difference between bi -quaternion and octonion formulations is that bi-quaternions are noncommutative but associative while the octonions are neither commutative nor associative and in split basis the role of associativity is played by the alternativity. Octonion has the advantage to work in higher dimensional space time. We may now discuss the decomposition of theories in terms of electric and magnetic charges in the following manner.

\subsection{1 (Electric Case)}

In this particular case (electric case) if we put that $V_{\mu}=V_{\mu}^{\star}$ i.e. $A_{\mu}-i B_{\mu}=A_{\mu}+i B_{\mu} \Rightarrow B_{\mu}=0$ or giving rise to $V_{\mu}=A_{\mu}$. Hence we get the following split octonion representation of covariant derivative in the absence of magnetic monopole i.e.

$$
D_{\mu} \rightarrow\left(\begin{array}{cc}
\partial_{\mu}+A_{\mu} & -A_{\mu}^{a} e_{a} \\
A_{\mu}^{a} e_{a} & \partial_{\mu}+A_{\mu}
\end{array}\right) ; \quad D_{\nu} \rightarrow\left(\begin{array}{cc}
\partial_{\nu}+A_{\nu} & -A_{\nu}^{a} e_{a} \\
A_{\nu}^{a} e_{a} & \partial_{\nu}+A_{\nu}
\end{array}\right)
$$

and then we get

$$
\left[D_{\mu}, D_{\nu}\right]=\left(\begin{array}{cc}
F_{\mu \nu} & -\overrightarrow{F_{\mu \nu}^{a}} \cdot \overrightarrow{e_{a}} \\
\overrightarrow{f_{\mu \nu}^{a}} \cdot \overrightarrow{e_{a}} & f_{\mu \nu}
\end{array}\right)=E_{\mu \nu}
$$

Consequently 


$$
D_{\mu} E_{\mu \nu}=\left(\begin{array}{cc}
j_{\nu} & -j_{\nu}^{a} e_{a} \\
j_{\nu}^{a} e_{a} & j_{\nu}
\end{array}\right)=J_{\nu}
$$

which is the split octonion form of generalized $U(1) \times S U(2)$ field equation where the diagonal elements represent the Maxwell's equation while the off diagonal elements describe the Yang Mills gauge fields in absence of magnetic monopole.

\subsection{2 (Magnetic Case)}

In this particular case (electric case) if we put that $V_{\mu}=-V_{\mu}^{*}$ i.e. $A_{\mu}-i B_{\mu}=-A_{\mu}-i B_{\mu} \Rightarrow$ $A_{\mu}=0 \Rightarrow V_{\mu}=-i B_{\mu}$ and hence $V_{\mu}^{\star}=i B_{\mu}$. Therefore, have

$$
\begin{aligned}
D_{\mu} & \rightarrow\left(\begin{array}{cc}
\partial_{\mu}-i B_{\mu} & i B_{\mu}^{a} e_{a} \\
i B_{\mu}^{a} e_{a} & \partial_{\mu}+i B_{\mu}
\end{array}\right), \\
D_{\nu} & \rightarrow\left(\begin{array}{cc}
\partial_{\nu}-i B_{\nu} & i B_{\nu}^{a} e_{a} \\
i B_{\nu}^{a} e_{a} & \partial_{\nu}+i B_{\nu}
\end{array}\right) .
\end{aligned}
$$

and

$$
\left[D_{\mu}, D_{\nu}\right]=\left(\begin{array}{cc}
F_{\mu \nu} & -\overrightarrow{F_{\mu \nu}^{a}} \cdot \overrightarrow{e_{a}} \\
\overrightarrow{f_{\mu \nu}^{a}} \cdot \overrightarrow{e_{a}} & f_{\mu \nu}
\end{array}\right)=H_{\mu \nu}
$$

and

$$
D_{\mu} H_{\mu \nu}=\left(\begin{array}{cc}
k_{\nu} & -k_{\nu}^{a} e_{a} \\
k_{\nu}^{a} e_{a} & k_{\nu}
\end{array}\right)=K_{\nu}
$$

which is the split octonionic form of generalized $U(1) \times S U(2)$ field equations where the diagonal elements represent the dual Maxwell equation i.e for pure magnetic monopole and off diagonal elements describe the Yang Mills gauge fields in in the absence of electric charge.

\section{Generalized Dirac Equations for Dyons}

We may now adopt the fore going analysis to obtain the Dirac equation for dyons on using the ES non - symmetric theory. The simplest free particle Dirac equation is given by

$$
\left(\gamma^{\mu} \partial_{\mu}+\kappa\right) \psi=0
$$

and to write the interacting form of Dirac equation one has to replace the partial derivative $\partial_{\mu}$ by covariant derivative $D_{\mu}$. So, we follow the same process and write the generalized Dirac equation for particles carrying electric and magnetic charges (i.e. dyons). Replacing the partial derivative $\partial_{\mu}$ by covariant derivative $D_{\mu}$, we may write following form of equation of a Dirac particle in generalized electromagnetic fields of dyons as 


$$
\left(\gamma^{\mu} D_{\mu}+\kappa\right) \psi=0
$$

where we have used the natural units of $c=\hbar=1$ and $D_{\mu}$ is covariant derivative in complex, quaternion and octonion tangent spaces of Einstein-Schrödinger non-symmetric theory. For complex case the covariant derivative is illustrated as

$$
D_{\mu} \quad \rightarrow \quad \partial_{\mu}+i q^{\star} V_{\mu}
$$

where

$$
q^{\star} V_{\mu} \rightarrow e A_{\mu}+g B_{\mu}
$$

Thus the Dirac equation is

$$
\left\{\gamma_{\mu}\left(\partial_{\mu}-i e A_{\mu}-i g B_{\mu}\right)+\kappa\right\} \psi=0
$$

or

$$
\left\{\gamma_{\mu} D_{\mu}+\kappa\right\} \psi=0
$$

which is invariant under gauge transformation as well. For quaternion case, we may write the covariant derivative as

$$
D_{\mu} \quad \rightarrow \quad \partial_{\mu}-i\left(q^{\star} V_{\mu}\right) e_{0}-\left(q^{\star a} V_{\mu}^{a}\right) e_{a}
$$

where

$$
q^{\star} V_{\mu} \rightarrow e A_{\mu}+g B_{\mu} ; \quad q^{\star a} V_{\mu}^{a} \rightarrow \varepsilon A_{\mu}^{a}+\varepsilon^{\prime} B_{\mu}^{a}
$$

where $\varepsilon$ and $\varepsilon^{\prime}$ are the Yang - Mill's coupling constants associated with the isotropic coupling parameters of electric and magnetic charges respectively. Similarly the Dirac equation for generalized fields of dyons in octonionic tangent space is described by equation (69) where the covariant derivative is given in the split octonion form as

$$
D_{\mu} \rightarrow\left(\begin{array}{cc}
\partial_{\mu}+q^{*} V_{\mu} & -q V_{\mu}^{a} e_{a} \\
q V_{\mu}^{* a} e_{a} & \partial_{\mu}+q V_{\mu}^{*}
\end{array}\right)
$$

which is double fold structure of quaternionic tangent space and is described in terms of Zorn's vector matrix realization of split octonion basis elements. 


\section{Discussion and Conclusion}

It is note worthy to include here, a motivation for the use of split octonion algebra instead of real octonion because the split octonion have the advantages to work in terms of matrix realizations while the due to non associativity of real octonions, it is impossible to write their correspondence with the matrix realizations. Secondly, the real octonion forms a metric in eight dimensional structure while the split octonion has the two $(4,4)$ (four fold ) degeneracy in complex space time and has the direct correspondence with the bi-quaternions. We may also develop a similar theory using the real octonion but in that case it will hard to give the four dimensional correspondence. So, this is why the quaternion-octonions play an important role in order to understand the physical theories of higher dimensional supersymmetry and super gravity etc. As we have mentioned that the octonions consist seven imaginary units resulting to seven permutations of $S U(2)$ Yang - Mill's fields. So we have the scope to enlarge the metric without putting the additional structure of space -time by hand and accordingly there is a possibility to define covariant derivative of a vector obtained in terms of octonionic vector potential and the octonion curvature. The automorphism group of octonion algebra is the 14- dimensional $G_{2}$ group [23, 24] which admits a $S U(3)$ sub-group and leaves the idempotent $u_{0}$ and $u_{0}^{\star}$ of split octonion algebra as invariant. We have established the connection between real and split basis of octonions and accordingly developed our present formulation. Due to the lack of associativity in octonion representation we have described octonion basis elements in terms of Zorn's vector matrix realizations where octonions are represented as the double fold degeneracy in terms of quaternion variables to maintain the consistency in our theory of dyonic fields. Equation (34) represents the non - symmetric metric in the complex tangent space for dyonic fields, where $k_{\mu \nu}$ is the anti - symmetric tensor associated with the generalized fields of dyons. Because the antisymmetric part has been described as further complex quantity our theory removes the conflicts that Maxwell tensor is real or imaginary and leaves all other good points of ES or NGT metric untouched. The Dyon field tensor is expressed by equations (35) and (36) in terms of electromagnetic field strengths associated with electric and magnetic sources. In this theory we have replaced the internal transformation $C_{\nu}$ by generalized gauge potential $V_{\nu}$ of dyons. It has been shown that the anti - symmetric part of the metric leads to generalized field equations of dyons discussed by equation (39). Accordingly, we have obtained the electric and magnetic field tensors from the generalized one for dyons as discussed by equation (40). Consequently, equation (41) describes the electric and magnetic four - currents obtained from the corresponding field tensors of dyons which are considered as the particles carrying simultaneous existence of electric and magnetic charges. Equations (42) to (44) are the unitary internal transformations for the dyonic gauge potential. Equation (46) represents the covariant derivative in the complex tangent space for dyonic fields, with the help of which we have obtained equations (47) and (48), which in fact represent the differential forms of generalized Maxwell's - Dirac equation for dyonic fields. Equation (49) expresses the covariant derivative for dyonic fields in the quaternionic tangent space of the non-symmetric theory in which the second term represents the electromagnetic part while the third term represents the non - Abelian part of Yang - Mill's field in terms of quaternion basis vectors. Also with the help of equation (49) we have obtained equation (50), which describes $U(1) \times S U(2)$ gauge structure of generalized quaternion tangent space. In equation (50) $G_{\mu \nu}$ and $G_{\mu \nu}^{a}$ are the gauge field strengths of Abelian and non - Abelian fields of dyons. In equation (52) $J_{\mu}$ and $J_{\mu}^{a}$ are generalized currents corresponding to the electromagnetic $U(1)$ part and non-Abelian $S U(2)$ part respectively for dyonic fields. Equations (53) and (54) represent the continuity equation where $J_{\mu}$ is expressed by equation (55) which in fact is the $U(1) \times S U(2)$ 
gauge structure of generalized current associated with dyons consisting point like electromagnetic $U(1)$ gauge structure having the four current $J_{\mu}$ followed by $S U(2)$ like extended Yang - Mill's gauge structure with non Abelian nature four current $J_{\mu}^{a}$. Equation (56) represents the split octonion derivative for dyonic fields in non - symmetric theory, which in fact is the double fold realization of quaternion derivative. With the help of equation (56) we have obtained equations (5758) and equation (59), respectively defines the double fold $U(1) \times S U(2)$ gauge structures of quaternion tangent space and generalized Dirac-Maxwell's equation for dyonic fields. Also equation (60) represents the continuity equation for dyonic fields in octonionic tangent space. It has been shown that the theory of dynamics of electric and magnetic charges is reproduced from the generalized theory of dyons using complex, quaternion and octonion tangent spaces. Equation (68) illustrates the covariant derivative for generalized fields of dyons in the complex tangent space of Einstein - Schrödinger non - symmetric theory, where $q^{\star} V_{\mu}$ is represented by equation (69). Consequently equation (70) is the Dirac equation for generalized fields of dyons in complex tangent space, which is invariant under gauge transformation and Lorentz transformation as well. Equation (71) represents the covariant derivative in quaternionic tangent space. In equation (72) $e$ and $g$ are electric and magnetic charges of dyons and $\varepsilon$ and $\varepsilon^{\prime}$ are Yang - Mill's coupling constants associated with the isotopic spin coupling parameters due to the presence of electric and magnetic charges respectively. Thus equation (73) represents the Dirac equation for generalized fields of dyons in quaternionic space of ES non - symmetric theory. Similarly equations (69) represents the Dirac equation for generalized fields of dyons in octonion tangent space if $D_{\mu}$ is described in its split octonion form given by equation (74). Here we see that the Dirac equation in the octonion tangent space is the doubly fold structure of quaternionic tangent space and is described in terms of Zorn's vector matrix realization of split octonion basis elements. As such, the fore going analysis describe the further extension of ES non symmetric metrics successfully and consistently in terms of three hyper complex number system namely complex, quaternion and octonion without imposing extra constrains. So, in nutshell, the present theory describes the combined gauge structures $G L(R) \otimes U(1)_{e} \otimes U(1)_{m} \otimes S U(2)_{e} \otimes S U(2)_{m}$ where $G L(R)$ describes Gravity, $U(1)_{e}$ demonstrates the electromagnetism due to the presence of electric charge, $U(1)_{m}$ is responsible for the electromagnetism due to magnetic monopole, $S U(2)_{e}$ demonstrates the Yang Mill's field due to the presence of electric charge while $S U(2)_{m}$ gives rise the another YangMills field due to the presence of magnetic monopole. It has also been shown that this unified picture reproduces the Gravity, electromagnetism and theory of Yang-Mill's field in the absence of magnetic monopole. Accordingly we have obtained the generalized Dirac equation for dyons from the covariant derivatives in terms of complex, quaternionic and octonionic tangent spaces.

Acknowledgment- The work is supported by Uttarakhand Council of Science and Technology, Dehradun. One of us OPSN is thankful to Chinese Academy of Sciences and Third world Academy of Sciences for awarding him CAS-TWAS visiting scholar fellowship to pursue a research program in China. He is also grateful to Professor Tianjun Li for his hospitality at Institute of Theoretical Physics, Beijing, China.

\section{References}

[1] A. Einstein, Rev. Modern Physics, 20 (1948), 35; Can. J. Math. $\underline{2}$ (1949), 120; The meaning of Relativity, (Princeton U.P., Princeton, NJ, 1955), Appendix 2; A. Einstein and E. G. Straus, Ann. Math., $\underline{\mathbf{4 7}}$ (1946), 731; A. Einstein and B. Kaufman, Ann. Math., $\underline{\mathbf{6 2}}$ (1955), 128. 
[2] E. Schrödinger, Proc. Royal Irish Acad. $\underline{\mathbf{A 5 1}}$ (1947), 163; $\underline{\mathbf{A 5 2}}$ (1948), 1; "Space-Time Structure", Cambridge Press, London, 1950.

[3] R. B. Mann and J. W. Moffat, J. Phys., A14 (1981), 2367; Phys. Rev., D26 (1982), 1858; G. Kunstatter, P. Leivo and P. Savaria, Class. and Quant. Grav., 1 (1984), 7.

[4] J. W. Moffat, Phys. Rev., D19 (1979), 3554; J. Math. Phys., 21 (1980), 1978; Phys. Rev., D35 (1987), 3733; "Non -symmetric Gravitational Theory", arXiv:gr-qc/9411006.

[5] J. W. Moffat and D. H. Boal, Phys. Rev., D11 (1975), 1375; W. B. Bonnor, Proc. Royal Soc. London Ser., $\underline{\mathbf{A 2 2 6}}$ (1954), 336; J. W. Moffat, J. Math. Phys., 25 (1984), 347; C.R.Johnson, Phys. Rev., D12 (1975), 3831.

[6] J. A. Shifflett, arXiv:gr-qc/0310124; gr-qc/0411016; gr-qc/0403052; gr-qc/0411016v4.

[7] K. Borchsenius. Phys. Rev., D13 (1976), 2707; J. W. Moffat and S. Okubo, Phys. Rev., D31 (1975), 1327.

[8] T. Damour, S. Deser and J. Mc Carthy; "Non -symmetric Gravity Theories: Inconsistencies and a Cure", arXiv:gr-qc/9207003 Phys. Rev., D47 (1993) 1541.

[9] T. Janssen and T. Prokopec, "Problems and hopes in non -symmetric gravity", eprint arXiv:gr-qc/0611005; J. A. Nieto and J. Socorro, "Is non -symmetric gravity related to string theory?", arXiv:hep-th/9610160.

[10] S. Morques and C. G. Oliveira, J. Math. Phys., $\underline{\mathbf{2 6}}$ (1985), 3131; Phys. Rev., D36 (1987), 1716.

[11] S. Marques- Bonham, J. Math. Phys., $\underline{\mathbf{2 9}}$ (1988), 2127; $\underline{\mathbf{3 1}}$, (1990), 1478; $\underline{\mathbf{3 2}}$, (1991), 1383;

[12] S. Ragusa, Class. Quant. Grav., 19 (2002), 5969; Phys. Rev., D62 (2000), 024026; D64 (2001), 029901 (erratum); D63 (2001),084019; D64 (2001), 067501.

[13] P. A. M. Dirac, Proc. Roy. Soc. London, $\underline{\mathbf{A 1 3 3}}$ (1931), 60; Phys. Rev., $\underline{\mathbf{7 4}}$ (1948), 817; G. 't Hooft, Nucl. Phys., B79 (1974), 276; A. M. Polyakov, JETP Lett., $\underline{\mathbf{2 0}}$ (1974) 194; K. Konishi, "The Magnetic Monopole Seventy-Five Years Later", arXiv:hep-th/0702102.

[14] J. Schwinger, Science, $\underline{\mathbf{1 6 5}}$ (1978), 757; Phys. Rev., $\underline{\mathbf{1 4 4}}$ (1986), 1087; $\underline{\mathbf{1 5 1}}$ (1966), 1048; $\underline{151}$ (1966), 1055; $\underline{173}$ (1968), 1536; D. Zwanziger, Phys. Rev., 176 (1968), 1489; Phys. Rev., D3 (1971), 880; B. Julia and A. Zee, Phys. Rev., D11 (1975), 2227.

[15] S. Mandelstem, Phys. Rev., D11 (1975), 3026; F. Röhrlich, Phys. Rev., 150 (1966), 1104. P. Goddard and D. I. Olive, Rep. Prog. Phys., $\underline{\mathbf{4 1}}$ (1978), 1357; J. A. Mignaco, Braz. J. Phys., $\underline{\mathbf{3 1}}$ (2001), 235.

[6] J. Preskill, "Magnetic Monopoles", Ann. Rev. of Nucl. and Part. Sci., $\underline{\mathbf{3 4}}$ (1984), 461; C. Dokos and T. Tomaras, Phys. Rev., D21 (1980), 2940; N. Craigie, "Theory and Detection of magnetic Monopoles in Gauge Theories", World Scientific, Singapur, 1986.

[17] B. S. Rajput and D. C. Joshi, Had. J., $\underline{4}$ (1981), 1805; Pramana (India) $\underline{15}$ (1980), 153; B. S. Rajput and Om Prakash, Indian J. Phys., $\underline{\mathbf{A 5 3}}$ (1979), 274.

[18] P. S. Bisht, O. P. S. Negi and B. S. Rajput, Prog.Theor. Phys., $\underline{\mathbf{8 5}}$ (1991), 157; IL Nuovo Cimento, $\underline{\mathbf{A 1 0 4}}$ (1991), 337; Inter. J. Theor. Phys., $\underline{\mathbf{3 2}}$ 1993), 2099. 
[19] P. S. Bisht, O. P. S. Negi and B. S. Rajput, Indian Journal of Pure and Appl. Maths, $\underline{\mathbf{2 4}}$ (1993), 543; Indian Journal of Pure and Appl. Phys, $\underline{\mathbf{2 8}}$ (1990), 157; $\underline{\mathbf{3 1}}$ (1993), 292; $\underline{\mathbf{3 2}}$ (1994), 184; $\underline{\mathbf{3 2}},(1994), 193$.

[20] Shalini Bisht, P. S. Bisht and O. P. S. Negi, IL Nuovo Cimento, B113, (1998), 1449; A. S. Rawat and O. P. S. Negi, IL Nuovo Cimento, $\underline{\mathbf{A 1 1 2}}$, (1999) 245; Jivan Singh, P. S. Bisht and O. P. S. Negi, J. Phys. A: Math. Theor., $\underline{40}$ (2007) 9137.

[21] N. Cabibo and E. Ferrari, IL Nuovo Cimento, $\underline{\mathbf{2 3}}$ (1962), 1147; A. Nisbet, Proc. Roy. Soc. London, $\underline{\mathbf{A 2 3 1}}$ (1955) 250.

[22] Shalini Dangwal, P.S.Bisht and O.P.S.Negi, "Octonionic Gauge Formulation for Dyonic Fields", arXiv:hep-th/0608061, ; "Unified Split Octonion Formulation of Dyons", arXiv: hep-th/0607209; P. S. Bisht, Bimal Pandey and O. P. S. Negi, 'Interpretations of Octonion Wave Equations", arXiv:0708.1664 [hep-th].

[23] M. Günaydin and F. Gursey, J. Math. Phys., 14 (1973), 1651; 17 (1976), 1875; Phys. Rev., D9 (1974), 3387; S. Catto, "Exceptional Projective Geometries and Internal Symmetries", arXiv:hep-th/0302079; J. C. Baez, Bull. Amer. Math. Soc., $\underline{39}$ (2001), 145; "The Octonions" , math. RA/0105155; M. Koca and N. Ozdes, J. Phys. A22, (1989), 1469; F.D.(Tony) Smith Jr., "Group and Standard Model with $130 \mathrm{GeV}$-truth quark from $D_{4}-D_{5}-D_{6}$ Model using $3 \times 3$ octonion matrices", arXiv; hep-th/9501252.

[24] C. Castro, J. Math. Phys., $\underline{\mathbf{4 8}}$ (2007), 073517; F. D. Smith Jr, Int. J. Theor. Phys., $\underline{\mathbf{2 4}}$ (1985), 155; $\underline{\mathbf{2 5}}$ (1985) , 355; P. M. Pavsic, Int. J. Mod. Phys., $\underline{\mathbf{A 2 1}}$ (2006), 5905; Phys. Lett., $\underline{\mathbf{B 6 1 4}}$, (2005) ,85. 